\title{
Length of Hospital Stay in Patients with Spinal Cord Injury
}

\author{
Hye Jin Jang, M.D., Jieung Park, M.D., Hyung-Ik Shin, M.D.
}

Department of Rehabilitation Medicine, Seoul National University College of Medicine, Seongnam 463-707, Korea

Objective To provide the latest statistics about the length of hospital stay (LOS) and the number of hospitals where the patient was admitted (NHA) for patients with spinal cord injury (SCI) and to investigate the correlated demographic characteristics.

Method In total, 277 patients with SCI who were members of the Korea Spinal Cord Injury Association were included in the analysis. The survey was conducted by self-completed questionnaires to collect data on LOS, NHA, and demographic variables.

Results Mean LOS was 13.5 \pm 9.7 months and the mean NHA was $2.7 \pm 1.4$. Patients who suffered from SCI by traffic accidents showed a longer LOS and larger NHA than those with other causes. The mean LOS for patients with traumatic SCI was longer than that whose cause of injury was disease. Patients discharged in the 2000s had a longer LOS and a larger NHA than those discharged earlier. Other factors such as gender, age at the time of injury, neurological category, and ambulation capability did not result in a significant difference in either LOS or NHA. Conclusion The mean LOS of domestic patients with SCI was longer than the values reported in foreign studies. Interestingly, neither neurological category nor functional status were related to LOS. These findings suggest that other factors such as socio-psychological factors, other than the medical state of the patient, have an effect on the LOS of patients with SCI in Korea.

Key Words Spinal cord injury, Length of stay, Hospitalization, Patient discharge

Received April 29, 2011; Accepted August 6, 2011

Corresponding author: Hyung-Ik Shin

Department of Rehabilitation Medicine, Bundang Seoul National University Hospital, 166, Gumi-ro, Bundang-gu, Seongnam 463-707, Korea

Tel: +82-31-787-7733, Fax: +82-31-787-4056, E-mail: hyungik1@snu.ac.kr (a) This is an open-access article distributed under the terms of the Creative Commons Attribution Non-Commercial License (http:// creativecommons.org/licenses/by-nc/3.0) which permits unrestricted noncommercial use, distribution, and reproduction in any medium, provided the original work is properly cited.

Copyright $\odot 2011$ by Korean Academy of Rehabilitation Medicine

\section{INTRODUCTION}

In cases of spinal cord injury (SCI), acute care is generally completed within a few months of injury and the neurological recovery reaches a plateau making it timely for the patient to be dischraged. ${ }^{1}$ However, in domestic cases, many patients seek several hospitals for admittance, instead of returning to ordinary life. A long hospital stay occupies medical resources and leads to substantial social loss. It also poses a heavy financial burden on the patient. ${ }^{2}$ The need to address problems caused by such a long hospital stay motivated this research on the length of hospital stay (LOS) of domestic patients with SCI and candidate related factors. 
A nationwide database on the number and characteristics of individuals with SCI is unavailable, because The Welfare of Disabled Persons Act in Korea does not recognize spinal cord impairment as an independent disability. Data collected by individual researchers are also very limited. According to an excerpt reported in 2002 by Lee et al. who surveyed 160 patients with SCI who had been admitted to the National Rehabilitation Center, the mean LOS was 13.7 months, and the mean number of hospitals where the patient was admitted (NHA) was 3.7. ${ }^{3} \mathrm{Kim}$ et al. surveyed patients who were staying in post-acute rehabilitation hospitals, and reported that the mean time since injury was 558 days, and the mean NHA was 2.25. ${ }^{4}$ These studies suggest that the mean LOS of domestic patients with SCI may be longer than that of patients in other countries. This is possible considering that the median number of hospitalized days for American patients with SCI is 12 days in acute care units and 38 days in rehabilitation units, and that the median duration of initial care is 133 days for patients with SCI in Australia. $^{5,6}$

However these domestic studies had some limitations. The study by Lee et al. was not formally published, and the detailed results have been lost. Besides, the surveyed subjects were patients discharged before the year 2000, which makes the results of the analysis outdated. Another flaw is that data were collected from patients treated at a particular hospital, instead of from a nationwide epidemiological census. ${ }^{3}$ The research by Kim et al. was also limited in that the statistics were summed over the patients who were not yet discharged at the time of the survey; thus, total LOS is unknown. ${ }^{4}$

Our study was conducted to provide statistics that reflect the current situation in the 2000s by investigating the LOS and related factors for subjects selected from a nationwide set of patients with SCI. The result of this research will have value as the first formal academic data on the LOS of domestic patients with SCI. These data are expected to be helpful when searching for efficient measures to provide medical services for patients with SCI in the future.

\section{MATERIALS AND METHODS}

\section{Subjects and procedure}

The survey was conducted using a questionnaire completed by patients with SCI registered with the Korea Spinal Cord Injury Association (KSCIA), a nonprofit civil organization. In particular, 11 municipal or provincial associations under the KSCIA first informed the research team of the number of patients with SCI available for survey. The research team accordingly sent questionnaires to each association. Each association mailed the questionnaires to the members with return envelopes and collected the completed questionnaires. They sent the collected questionnaires to the research team with private information excluded.

\section{Survey items}

Preliminary questionnaires were distributed to 20 patients with SCI to check the suitability of the questions. This preliminary survey and a group discussion revised the preliminary questionnaire into the final version after one revision. The completed questionnaire consisted of three questions on key information, and five questions on basic information.

Patients were requested to indicate the date of injury, the date of final discharge to home, and the names of all hospitals where they had been admitted. The purpose of these questions was to collect data on LOS and NHA. They were not directly asked to enhance the credibility of the data. Instead, the LOS was measured at the level of month from the date of admission and date of discharge, whereas the NHA was counted from the list of hospitals where the patient had been admitted. The preliminary survey disclosed that the patients recalled the dates of injury and discharge to the level of month well and the names of all hospitals where they had been admitted.

For basic information, questions were asked on gender, birth date, neurological category of SCI, cause of injury, ambulation capability, and use of assistive devices. Age at the time of injury was calculated from the responses to the questions about the date of birth and the date of injury. For the question about neurological category, the patients chose one from among the following items: complete paraplegia, incomplete paraplegia, complete tetraplegia, incomplete tetraplegia, others, and don't know. A choice of trauma or disease was given for the cause of injury. Those who chose trauma were requested to make further choices from among traffic accident (TA), falls, diving accident, other sport injuries, violence and others. Those who chose disease chose again from 
among spinal cord tumor, myelitis congenital disorders, spinal tuberculosis, spinal stenosis, or other degenerative changes of the spine, and others. For the information on capability of ambulation and use of assistive devices, a question was prepared requesting a selection from 1) "cannot walk at all", 2) "walk a few steps at home using assistive devices (lower extremity orthosis, cane, crutch etc.)", 3) "walk at home without assistive devices", 4) "walk outdoors using assistive devices", 5) "walk outdoors without using assistive devices" and 6) "other". Those who chose item 1) were classified into a "capable to walk' group, and those who chose 2), 3), 4) or 5) were classified into an "incapable to walk" group, whereas those who responded with 6 ) were classified into the "unknown ambulatory capability" group.

\section{Data analysis}

In total, 475 questionnaires were collected of the 610 distributed. The questionnaires with blank answers to one of the three questions (date of injury, date of discharge, list of admission hospitals), which are indispensible to measure LOS and NHA, were discarded. Responses that did not supply basic information such as date of birth and gender or did not answer more than three questions were also excluded for fear that too many unanswered questions would suggest the insincerity of response. Finally, 277 responses remained for analysis.

SPSS 18.0 (SPSS Inc., Chicago, USA) was used for the statistical analysis. Mean LOS and NHA were calculated for the entire sample. Descriptive analyses were applied to the demographic factors, and $t$-tests were conducted to infer differences in the mean LOS and NHA between the two groups classified by factors such as gender (male vs. female), cause of injury (trauma vs. disease), and ambulation capability (capable vs. incapable). The "age at the time of injury" factor generated five groups, and "time of discharge" grouped the patients into three period categories (before the 1990s, during the 1990s, and in the 2000s), whereas the SCI neurological category provided three groups. A one-way analysis of variance was applied for each demographic factor that generated more than three groups. Multiple regression analyses were also conducted to identify factors with an effect on either LOS or NHA. Independent variables included gender, age at the time of injury, neurological category of injury, cause of injury, capability of ambulation, and time of discharge.
The estimated regression model was finally derived using the stepwise method of variable selection. The critical p-value to conclude a statistically significant difference was set at 0.05 .

\section{RESULTS}

\section{Demographic characteristics}

The analyzed sample was composed of 218 males (78.7\%) and 59 females $(21.3 \%)$. Age at the time of injury showed that 37 subjects $(13.4 \%)$ were $<20$ years, 106 (38.3\%) were in their 20s, $91(32.9 \%)$ in their 30s, $30(10.8 \%)$ in their 40 s, and $13(4.7 \%) \geq 50$ years, with a mean of 30.0 years and a median of 29 years. When counted by neurological category, 150 patients $(54.2 \%)$ had complete paraplegia, $34(12.3 \%)$ had complete tetraplegia, 80 (28.9\%) had incomplete paralysis, and $13(4.7 \%)$ had an unknown neurological injury category. The cause of injury showed that 251 subjects $(90.6 \%)$ had suffered a traumatic injury, whereas disease caused SCI in 16 subjects (5.8\%) and the cause was unknown in 10 subjects (3.6\%). Among the subjects with a known cause, 134 (48.4\% of the whole sample) had a TA cause, whereas $133(48.0 \%)$ had other causes. Forty-nine subjects $(17.7 \%)$ were discharged before the 1990s, 117 (42.2\%) during the 1990s, and 111 (40.1\%) in the 2000s. Finally, 235 patients $(84.8 \%)$ were incapable of ambulation and $35(12.6 \%)$ were capable. Ambulatory capability could not be assessed in seven patients (2.5\%) (Table 1).

\section{Mean LOS and NHA}

Mean LOS was 13.5 \pm 9.7 months, with a median of 10.2 months, as estimated from the entire sample of 277 subjects. Mean NHA was $2.7 \pm 1.4$ with a median of 3 (Table $1)$.

\section{Mean LOS and NHA by demographic characteristics}

Cause of injury: Mean LOS was $13.9 \pm 9.8$ months for patients with traumatic SCI, which was significantly longer than $8.2 \pm 8.0$ months as the estimate for those with a disease cause $(\mathrm{p}=0.022)$. The mean NHA was $2.7 \pm 1.4$ for traumatic patients, whereas it was $2.5 \pm 1.7$ for those with a disease cause, resulting in a significant difference (Fig. 1).

Mean LOS was $15.7 \pm 10.2$ months for subjects with TA as a cause, which was significantly longer than $11.5 \pm 8.8$ 
Table 1. Length of Hospital Stay and Number of Hospitals Where the Patient was Admitted after Injury

\begin{tabular}{|c|c|c|c|c|}
\hline & Subgroup & (n) & Length of stay months & Number of hospitals \\
\hline $\begin{array}{l}\text { Total } \\
\text { (Median) }\end{array}$ & & 277 & $\begin{array}{c}13.5 \pm 9.7 \\
(10.2)\end{array}$ & $\begin{array}{c}2.7 \pm 1.4 \\
(3)\end{array}$ \\
\hline \multirow[t]{2}{*}{ Etiology (1) } & Trauma & 251 & $13.9 \pm 9.8$ & $2.7 \pm 1.4$ \\
\hline & Disease & 16 & $8.2 \pm 8.0$ & $2.5 \pm 1.7$ \\
\hline \multirow[t]{2}{*}{ Etiology (2) } & $\mathrm{TA}$ & 134 & $15.7 \pm 10.2$ & $2.9 \pm 1.3$ \\
\hline & Other than TA & 133 & $11.5 \pm 8.8$ & $2.5 \pm 1.5$ \\
\hline \multirow[t]{3}{*}{ Neurologic category } & Complete paraplegia & 150 & $13.4 \pm 9.7$ & $2.5 \pm 1.3$ \\
\hline & Complete tetraplegia & 34 & $14.1 \pm 8.3$ & $2.8 \pm 1.3$ \\
\hline & Incomplete injury & 80 & $13.6 \pm 10.5$ & $2.9 \pm 1.6$ \\
\hline \multirow[t]{2}{*}{ Ambulation } & Unambulatory & 235 & $13.6 \pm 9.6$ & $2.7 \pm 1.4$ \\
\hline & Ambulatory & 35 & $12.8 \pm 9.5$ & $2.7 \pm 1.5$ \\
\hline \multirow[t]{3}{*}{ Discharge } & -1989 & 49 & $9.4 \pm 8.1$ & $2.0 \pm 0.9$ \\
\hline & 1990-1999 & 117 & $12.2 \pm 8.3$ & $2.3 \pm 1.1$ \\
\hline & $2000-$ & 111 & $16.6 \pm 10.8$ & $3.4 \pm 1.5$ \\
\hline \multirow[t]{2}{*}{ Gender } & Male & 218 & $13.5 \pm 9.1$ & $2.7 \pm 1.3$ \\
\hline & Female & 59 & $13.4 \pm 11.6$ & $2.7 \pm 1.7$ \\
\hline \multirow[t]{5}{*}{ Age of injury } & $<20$ & 37 & $12.2 \pm 8.8$ & $2.7 \pm 1.2$ \\
\hline & $20-29$ & 106 & $12.2 \pm 9.0$ & $2.5 \pm 1.3$ \\
\hline & $30-39$ & 91 & $14.4 \pm 9.5$ & $2.8 \pm 1.4$ \\
\hline & $40-49$ & 30 & $16.9 \pm 12.3$ & $2.9 \pm 1.6$ \\
\hline & $50 \leq$ & 13 & $12.3 \pm 11.1$ & $2.9 \pm 1.7$ \\
\hline
\end{tabular}

Values are mean and standard deviation.

TA: Traffic accident

(A)

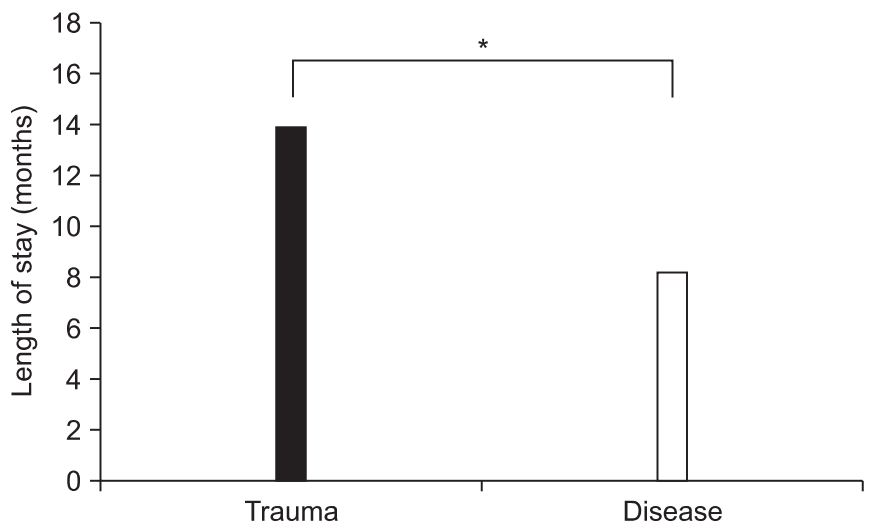

(B)

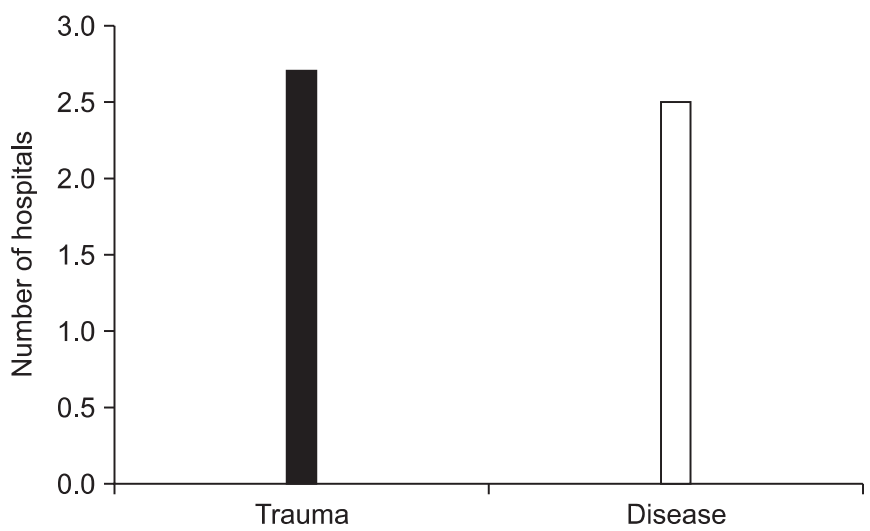

Fig. 1. Comparison of length of hospital stay and number of hospitals where the patient was admitted after injury between the trauma and disease group. (A) The trauma group showed a significantly longer hospital stay than that in the disease group. (B) The number of hospitals in the trauma group tended to be larger than that in the disease group. ${ }^{*} \mathrm{p}<0.05$.

months for cases with other causes $(\mathrm{p}=0.000)$. The mean NHA was $2.9 \pm 1.3$ for TA cases, which was significantly longer than $2.5 \pm 1.5$ cases with other causes ( $\mathrm{p}=0.042$ ) (Fig.
2).

Neurological category: Mean LOS was $14.1 \pm 8.3$ months for complete tetraplegia cases, $13.4 \pm 9.7$ months for 
(A)

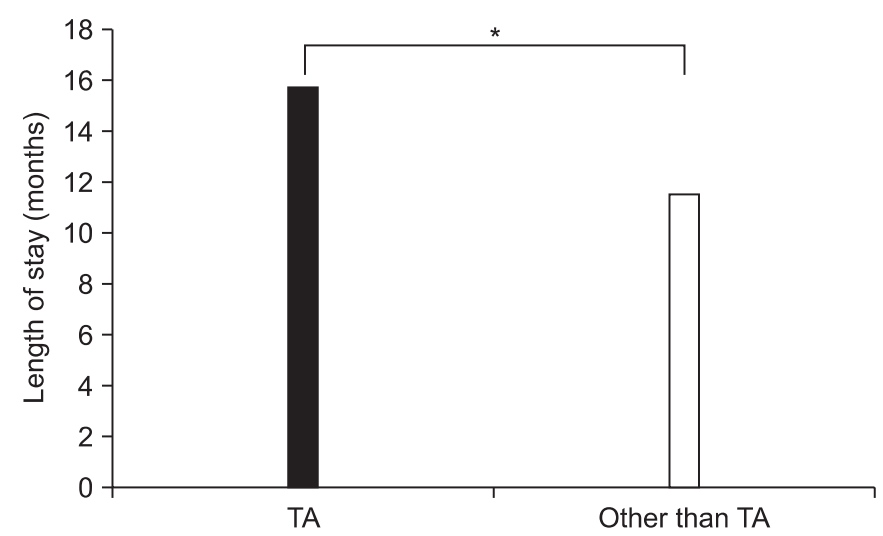

(B)

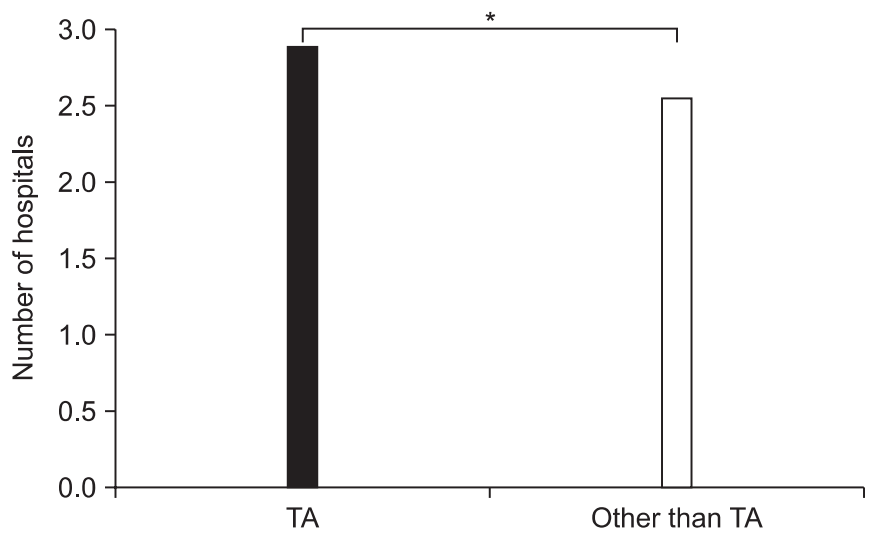

Fig. 2. Comparison of length of hospital stay and number of hospitals where the patient was admitted after injury between the traffic accident and other etiology groups. (A) The traffic accident group showed a significantly longer hospital stay than that of the other etiology group. (B) The traffic accident group was admitted to a significantly larger number of hospitals than that of the other etiology group. * $\mathrm{p}<0.05$, TA: Traffic accident.

(A)

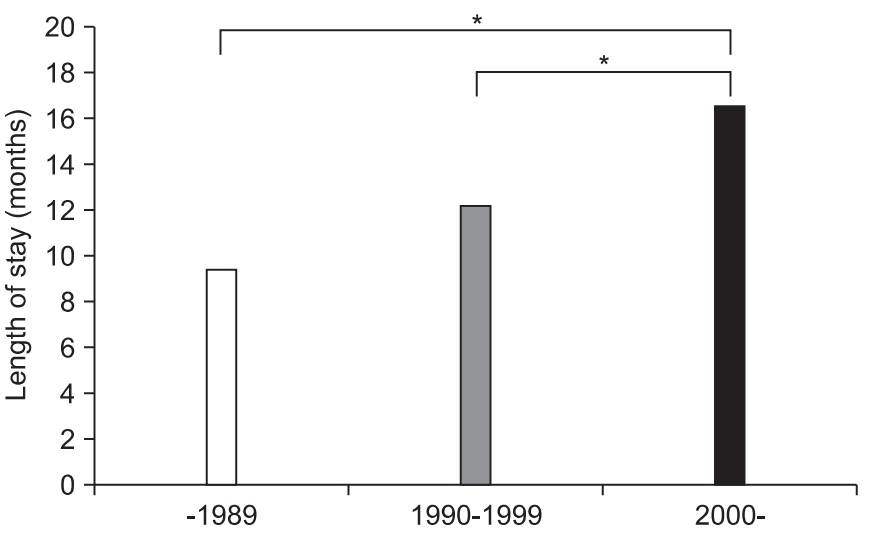

(B)

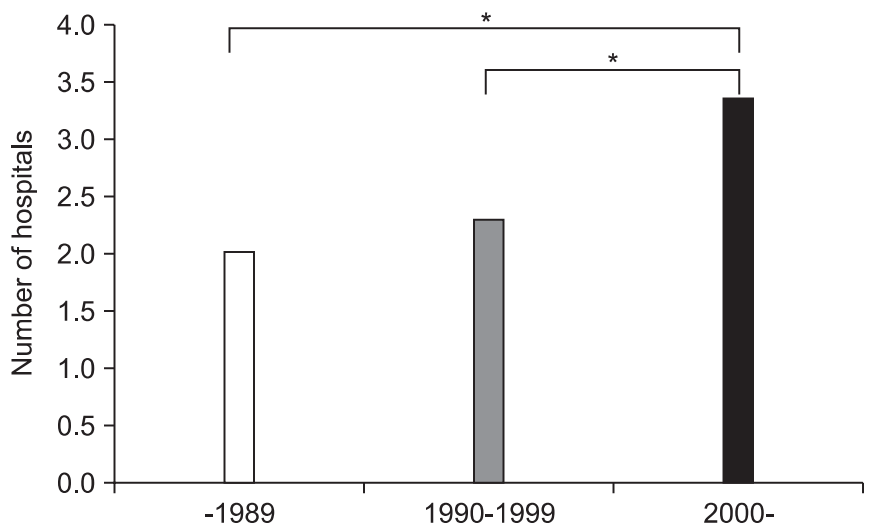

Fig. 3. Comparison of length of hospital stay and number of hospitals where the patient was admitted after injury among three groups classified by point of discharge. (A) Patients who were discharged in 2000 and later showed significantly longer hospital stays than patients who were discharged before 1990, and patients who were discharged from 1990-1999, respectively. (B) Patients who were discharged in 2000 and later showed that they were admitted to a significantly larger number of hospitals than patients who were discharged before 1990 and patients who were discharged from 1990-1999, respectively. *p<0.05.

complete paraplegia cases, and 13.6 \pm 10.5 months for incomplete injuries. But the differences among these three categories were not statistically significant. The mean NHA was $2.8 \pm 1.3$ for complete tetraplegia, $2.5 \pm 1.3$ for complete paraplegia, and $2.9 \pm 1.6$ for incomplete injuries, which was not statistically significant (Table 1).

Functional status: For those incapable of ambulation, the mean LOS was $13.6 \pm 9.6$ months, and the mean NHA was $2.7 \pm 1.4$. For those capable of ambulation, the mean LOS was $12.8 \pm 1.6$ months, and the mean NHA was $2.7 \pm$
1.5, but the differences were not statistically significant (Table 1).

Time of discharge: Mean LOS was $16.6 \pm 10.8$ months for subjects discharged in the 2000s, which was significantly longer than 12.2 \pm 8.3 months for those discharged in the 1990s and 9.4 \pm 8.1 months for those discharged before the 1990s ( $\mathrm{p}=0.000)$. Similarly, the mean NHA was $3.4 \pm 1.5$ for those discharged in the 2000s, which was significantly greater than $2.3 \pm 1.1$ for those discharged in the 1990s and $2.0 \pm 0.9$ for those discharged before the $1990 \mathrm{~s}(\mathrm{p}=0.000)$ 
Table 2. Multiple Regression Analysis for Length of Hospital Stay and Number of Hospitals Where the Patient was Admitted after Injury

\begin{tabular}{lcc}
\hline \multicolumn{1}{|c}{ Variable } & $\begin{array}{c}\text { Unstandardized } \\
\text { coefficients (B) }\end{array}$ & p-value \\
\hline $\begin{array}{l}\text { Length of stay } \\
\text { Constant }\end{array}$ & 14.437 & \\
\hline Traffic accident & 3.718 & 0.002 \\
\hline Point of discharge & & \\
\hline-1989 & -6.029 & 0.001 \\
\hline 1990-1999 & -3.997 & 0.002 \\
\hline 2000- (reference group) & & \\
Number of hospitals & & \\
\hline Constant & 3.373 & \\
\hline Point of discharge & & \\
\hline -1989 & -1.348 & 0.000 \\
\hline 1990-1999 & -1.071 & 0.000 \\
\hline 2000- (reference group) & & \\
\hline
\end{tabular}

(Fig. 3).

Other demographic factors: Gender and age at the time of injury did not result in any statistically significant differences in LOS or NHA (Table 1).

Multiple regression analysis: Multiple regression analyses were conducted on the variables that resulted in significant differences in either LOS or NHA. The estimated regression model demonstrated that time of discharge and presence of TA were factors associated with the significant differences in LOS (Table 2). Gender, age at the time of injury, SCI neurological category, cause of injury (trauma or disease), and ambulation capability were insignificant factors for LOS.

A multiple regression analysis on NHA disclosed that the time of discharge was the sole significant factor (Table 2). Gender, age at the time of injury, SCI neurological category, cause of injury, and ambulation capability were insignificant.

\section{DISCUSSION}

This is the first academic report on the LOS of Korean patients with SCI. Because the survey was conducted on a sample from a nationwide set of patients with SCI, it adds to the significance. The analysis conducted on data collected from 277 patients with SCI in various local communities disclosed that the mean LOS was 13.5 months and the mean NHA was 2.7.

In contrast to domestic cases, the median LOS of American patients with SCI is 12 days in acute care units and 38 days in rehabilitation units, as shown in a report released by the American National Spinal Cord Injury Statistical Center in 2010. It was also reported that $87.7 \%$ of the patients were discharged to the place of preinjury residence. ${ }^{5}$ In Australia, the median duration of initial care was 133 days for patients who were discharged in 2007 and 2008, according to statistics published by Australian Institute of Health and Welfare in $2010{ }^{6}$ Surveys conducted on patients in Europe who were admitted into eight rehabilitation centers in the Netherlands from 2000-2003 revealed that the mean LOS was 212-387 days depending on injury severity. ${ }^{7}$ The median LOS of the patients with traumatic SCI who were admitted to 32 rehabilitation centers in Italy from 19971999 was 122 days. $^{8}$ The mean LOS in Japan is 288 days for patients with SCI who underwent rehabilitation therapy at Kanagawa Rehabilitation Hospital, according to statistics published in $1994 .{ }^{9}$ Thus, comparing the results of this research with foreign statistics shows that the LOS of Korean patients with SCI is longer than that found in other countries. It also indicates that domestic patients with SCI seek 2.7 hospitals for admittance, whereas most patients in other countries are discharged directly to home. ${ }^{5}$

In contrast, the LOS of domestic patients with SCI was conspicuously longer than that of domestic patients with stroke. Surveys on patients with acute stroke admitted into university hospitals in Korea have reported that the mean LOS is 19.2-26.8 days. ${ }^{10,11}$ These statistics only reflect the length of stay in individual hospitals; thus, it is not valid to compare them directly with the results disclosed in the present study. However, Kim et al., who surveyed at-home patients with stroke, excluding those who had never been admitted to a hospital or received only acute-phase therapy, reported a mean LOS of 234.3 days, which was smaller than that reported here. ${ }^{12}$ This coincides with the research results of Lee et al. who concluded that the LOS of patients with SCI was longer than that of patients with stroke after surveying patients who had been discharged from in-patient rehabilitation at university hospitals. ${ }^{13}$

The LOS is often thought of as an indicator of the 
efficiency for providing medical services. This line of thinking is rooted in the assumption that efficient management of medical resources leads to identical outcomes in a shorter span of time. However, the LOS is actually influenced by many factors such as disease severity, patient insurance compensation plan, social resources available to the patient, psychological state of the patient, and others. ${ }^{1}$ According to reports in the USA, Italy, and Australia, the LOS is longer for patients with tetraplegia than those with paraplegia, and longer for those with a complete injury than those with an incomplete injury. ${ }^{1,6,8,13,14}$ Furthermore, the mean LOS is longer for patients with more severe functional impairments at the time of admission, for those who suffered a longer delay between injury and admission, for those discharged in the remote past, for those of younger ages, and for those with a higher education level. The LOS also tends to be long when a patient suffers from another disease or accompanying injuries or needs the assistance of devices such as a urinary catheter, receives surgical intervention, or is affected by complications such as bedsores. ${ }^{1,6,8}$

However, our results showed no significant differences in LOS across neurological SCI categories. Ambulation capability, which reflects a patient's functional status also did not significantly influence LOS. In other words, medical states or functional status were insignificant factors for the LOS in domestic patients, contrary to the findings of studies in other countries.

The LOS of patients with a traumatic cause was significantly longer than the LOS for whom disease was the cause. Similar conclusions were drawn in studies in the USA, which categorized patients into traumatic and non-traumatic groups by the cause of injury. ${ }^{15}$ Studies conducted in Israel and Italy also produced the same conclusions. ${ }^{16,17}$ It has been suggested that such a long LOS for traumatic patients may be due to the fact that they need a longer hospital stay because they tend to have more serious neurological damage or are affected with associated traumatic injuries or medical complications. Furthermore, the rehabilitation goal is set higher for them, because they have a longer life expectancy compared to patients with non-traumatic SCI. ${ }^{15-17}$

We also compared patients with a TA cause and those with other causes. T-tests showed that the LOS and the
NHA of the TA cause group were significantly longer than those with other causes. Sohn at al. reported a similar result after surveying patients who had been admitted into rehabilitation departments. They reported that the rate of hospital transfer was higher than the rate of discharge to home for patients who benefitted from automobile insurance or industrial-accident compensation insurance, compared to that of patients covered by medical insurance. ${ }^{18}$ They indicated that this tendency was caused by patient out-of-pocket medical expenses regardless of their functional state. We also conjectured that patients with SCI caused by TAs tended to stay longer in the hospital due to their light financial burden, but we could not reach this conclusion, because we did not investigate the medical expense payment mode.

The group of patients discharged in the 2000s showed longer LOS and larger NHA than those discharged in the 1990s and those discharged before the 1990s. This observation is contrary to the trend that the mean LOS of American patients with SCI tends to get shorter as time advances. ${ }^{1,5,19}$ This tendency could be explained by changes in the domestic medical environment. In Korea, rehabilitation medicine was introduced in the mid-1980s and took time for to get established. Patients with SCI were often left uncared for at home after the minimal acute-phase treatment in the days when rehabilitation treatment was not generally accessible. It could be construed that the recent increase in the number of rehabilitation centers has increased demand for rehabilitation treatment, which lengthened the LOS of the patients. The population characteristics may have acted as a source of bias to change the LOS across the periods categorized by time of discharge. Because a TA is a factor that can produce a significant difference in the LOS, it seemed reasonable to assume the hypothesis that the LOS tended to increase across time period categories, as the proportion of patients with TA in KSCIA increased over time. However, the "time of discharge" factor was significant for LOS and the NHA even after adjusting for the presence of TA in the multiple regression analysis. This result indicates that the change in the mean LOS across the time period was not solely due to the bias rendered by the presence of TA but showed a real trend.

Other reasons for the long hospital stay of domestic patients with SCI could be the lack rehabilitation and 
welfare facilities that help discharged patients with SCI restore themselves to their everyday lives and the difficulties of patient mobility, which are aggravated by Korean house structure..$^{20}$ Additionally, the fact that discharge is delayed for patients to plan and prepare for practical lives, because they lose self esteem after the injury and they are slow in recognizing and accepting that their functional impairments could be counted as possible reasons for a long hospital stay. ${ }^{4,21}$ The conclusion that the LOS of domestic patients with SCI is poorly related to SCI neurological category or functional status, suggests that the aforementioned socio-psychological factors may be more important in determining the LOS of domestic patients. In this context, more effort should be focused on providing more consultation and training to restore school or work, to supply disabled transportation, and to provide a subsidy for house remodeling aimed at improving patient mobility. Professional psychotherapy and peer consultation or self-assistance associations would be of much help for cases of low self esteem. ${ }^{21}$ According to the report by Kim et al., patients want to extend their hospital stay with the unfounded hope for further neurological recovery even after it reaches a plateau. This problem could be addressed with education or a publicity campaign about the feasible goals of rehabilitation therapy after SCI, leading to a shorter mean LOS. ${ }^{4}$

The main purpose of this study was to collect data on the LOS of domestic patients with SCI. Therefore, only five questions, which were not directly related to LOS or NHA, were included in the questionnaire to enhance the response rate. As a result, such factors as the presence of complications during a hospital stay and the history of surgical intervention, which are probable factors for LOS, remained beyond the scope of analysis.

Although the current study surveyed patients with SCI sampled from a nationwide population, the questionnaires were distributed only to members of certain disability associations. Due to this flaw, there is a limit to generalize the findings of the present study. For example, members enrolled in a disabilities association may be more active in social activities than non-members and this tendency may have exerted some influence on the LOS. A good alternative would be to establish a nationwide database through a SCI model system, similar to the one in the US, and to publish census statistics periodically.

\section{CONCLUSION}

A survey on 277 subjects selected from a nationwide set of patients with SCI resulted in a mean LOS of 13.5 months and an NHA of 2.7, which was longer than the estimates observed in other countries. Cause of injury and time of discharge were correlated with LOS, whereas such factors as neurological category, ambulation capability, gender, and age at the time of injury were not.

The finding that the neurological injury category and ambulation capability did not affect LOS may indicate that the long hospital stay of domestic patients with SCI is affected by such socio-psychological factors as deficient services, limited mobility, poor social rehabilitation and welfare infrastructure, and delayed acquisition of a sense of identity as a disabled individual, than by medical or functional factors. Therefore, to encourage patients to re-enter society, more effort should be focused on establishing rehabilitation services in local communities, subsidy programs to procure disabled transportation and house remodeling, education on the recovery process and the rehabilitation goal, and psychological support from others.

\section{REFERENCES}

1. Eastwood EA, Hagglund KJ, Ragnarsson KT, Gordon WA, Marino RJ. Medical rehabilitation length of stay and outcomes for persons with traumatic spinal cord injury: 1990-1997. Arch Phys Med Rehabil 1999; 80: 1457-1463

2. Lee KS, Bae HJ, Kim HS. Utilization of health care resources and costs of stroke patients: patients' perspective. J Korean Neurol Assoc 2004; 22: 583-589

3. Lee BS, Shin HI, Jang SJ, Kim BS. The hospital days and factors influencing on hospital days in patients with spinal cord injury. Proceedings of the 30th Annual Conference of The Korean Academy of Rehabilitation Medicine; 2002 Oct 17-18; Seoul, Korea: Medrang; 2002

4. Kim WS, Kang EK, Shin HI, Bang MS, Shin JC, Park JW. Desire for rehabilitation services of patients with spinal cord injury admitted in post-acute 
rehabilitation facilities. J Korean Acad Rehab Med 2008; 32: 169-174

5. National Spinal Cord Injury Statistical Center. 2010 Annual statistical report for the spinal cord injury model systems. Alabama: National Spinal Cord Injury Statistical Center; 2010, 17-18

6. Norton L. Spinal cord injury Australia 2007-08, Canberra: Australian Institute of Health and Welfare; 2010, 15-16

7. de Groot S, Dallmeijer AJ, Post MW, van Asbeck FW, Nene AV, Angenot EL, van der Woude LH. Demographics of the Dutch multicenter prospective cohort study 'Restoration of mobility in spinal cord injury rehabilitation'. Spinal Cord 2006; 44: 668-675

8. Pagliacci MC, Celani MG, Zampolini M, Spizzichino L, Franceschini M, Baratta S, Finali G, Gatta G, Perdon L. An Italian survey of traumatic spinal cord injury. The Gruppo Italiano Studio Epidemiologico Mielolesioni study. Arch Phys Med Rehabil 2003; 84: 1266-1275

9. Hasegawa $\mathrm{Y}$, Ohashi M, Ando N, Hayashi T, Ishidoh T, Kumagai K, Shimazu A. Spinal cord injury hospitalisation in a rehabilitation hospital in Japan. Paraplegia 1994; 32: 47-51

10. Chang H, Yoon SS, Kwon YD. Determinants of inpatient charges of acute stroke patients in two academic hospitals: comparison of intracerebral hemorrhage and cerebral infarction. J Korean Neurol Assoc 2009; 27: 215-222

11. Cho SC, Choi KH, Lee DJ, Ha SB. Interval between initial hospital arrival and start of rehabilitation therapy in sroke patients of tertiary medical center. J Korean Acad Rehab Med 1998; 22: 9-14

12. Kim WH, Lee KB, Kim SK, Jang SN, Kim BS, Lim HJ, Kim SO. Utilization status of rehabilitation hospital or clinic in patients with stroke. Korean J Stroke 2005; 7: 144-150
13. Rhie KS, Rah UW, Lee IY, Yim SY, Kim KM, Moon DJ, Lee JB. The discharge destination of rehabilitation inpatients in a tertiary hospital. J Korean Acad Rehab Med 2005; 29: 135-140

14. Tooth L, McKenna K, Geraghty T. Rehabilitation outcomes in traumatic spinal cord injury in Australia: functional status, length of stay and discharge setting. Spinal Cord 2003; 41: 220-230

15. McKinley WO, Seel RT, Gadi RK, Tewksbury MA. Nontraumatic vs. traumatic spinal cord injury: a rehabilitation outcome comparison. Am J Phys Med Rehabil 2001; 80: 693-699

16. Ronen J, Itzkovich M, Bluvshtein V, Thaleisnik M, Goldin D, Gelernter I, David R, Gepstein R, Catz A. Length of stay in hospital following spinal cord lesions in Israel. Spinal Cord 2004; 42: 353-358

17. Celani MG, Spizzichino L, Ricci S, Zampolini M, Franceschini M. Spinal cord injury in Italy: a multicenter retrospective study. Arch Phys Med Rehabil 2001; 82: 589-596

18. Sohn MK, Cho KH, Kim BO, Han SM. Discharge destinations after acute rehabilitation care. J Korean Acad Rehab Med 2003; 27: 269-274

19. Bryce TN, Ragnarsson KT, Stein AB. Spinal cord injury. In: Braddom RL, Editor. Physical medicine and rehabilitation, 3rd ed, Philadelphia, Saunders, 2007, 1287-1288

20. Bae CH, Seo DM. A phenomenological study on life of persons with spinal cord injury - difficulties in social rehabilitation. Journal of Rehabilitation Research 2008; 12: 117-141

21. Shin EK, Choi JA. Study on factors affecting on the spinal cord injuries' social integration using the structural equation model: analyzing mediating effect of depression, disability identity. Journal of Rehabilitation Research 2007; 11: 1-29 